\title{
Clusters of Drug-Resistant Mycobacterium tuberculosis Detected by Whole-Genome Sequence Analysis of Nationwide Sample, Thailand, 2014-2017
}

\author{
Ditthawat Nonghanphithak, Angkana Chaiprasert, Saijai Smithtikarn, Phalin Kamolwat, \\ Petchawan Pungrassami, Virasakdi Chongsuvivatwong, Surakameth Mahasirimongkol, \\ Wipa Reechaipichitkul, Chaniya Leepiyasakulchai, Jody E. Phelan, David Blair, Taane G. Clark, Kiatichai Faksri
}

Multidrug-resistant tuberculosis (MDR TB), pre-extensively drug-resistant tuberculosis (pre-XDR TB), and extensively drug-resistant tuberculosis (XDR TB) complicate disease control. We analyzed whole-genome sequence data for 579 phenotypically drug-resistant $M$. tuberculosis isolates (28\% of available MDR/pre-XDR and all culturable XDR TB isolates collected in Thailand during 2014-2017). Most isolates were from lineage $2(n=482 ; 83.2 \%)$. Cluster analysis revealed that $281 / 579$ isolates (48.5\%) formed 89 clusters, including 205 MDR TB, 46 pre-XDR TB, 19 XDR TB, and 11 poly-drug-resistant TB isolates based on genotypic drug resistance. Members of most clusters had the same subset of drug resistance-associated mutations, supporting potential primary resistance in MDR TB ( $\mathrm{n}=$ $176 / 205 ; 85.9 \%$ ), pre-XDR TB ( $n=29 / 46 ; 63.0 \%$ ), and XDR TB ( $n=14 / 19 ; 73.7 \%)$. Thirteen major clades were significantly associated with geography $(p<0.001)$. Clusters of clonal origin contribute greatly to the high prevalence of drug-resistant TB in Thailand.

7 uberculosis (TB), caused by Mycobacterium tuber1 culosis, is a major global public health issue. Southeast Asia contributes notably (44\%) to global TB cases.

Author affiliations: Khon Kaen University, Khon Kaen, Thailand (D. Nonghanphithak, W. Reechaipichitkul, K. Faksri); Mahidol University, Bangkok, Thailand (A. Chaiprasert, C. Leepiyasakulchai); Ministry of Public Health, Bangkok (S. Smithtikarn, P. Kamolwat, P. Pungrassami); Prince of Songkla University, Hat Yai, Songkhla, Thailand (V. Chongsuvivatwong); Ministry of Public Health, Nonthaburi, Thailand (S. Mahasirimongkol); London School of Hygiene and Tropical Medicine, London, UK (J.E. Phelan, T.G. Clark); James Cook University, Townsville, Queensland, Australia (D. Blair)

DOI: https://doi.org/10.3201/eid2703.204364
Thailand is in the top 30 countries for drug-resistant (DR) TB incidence (1). DR TB, including rifampinresistant TB and strains with additional resistance to isoniazid (multidrug-resistant [MDR] TB), remains a great challenge for TB control. In 2018, $\approx 500,000$ new cases of rifampin-resistant TB were reported globally, of which $78 \%$ were MDR TB (1). More worrisome is extensively drug-resistant (XDR) TB, which further exhibits resistance to 1 fluoroquinolone and 1 injectable second-line drug. The average proportion of global MDR TB cases with XDR TB is $6.2 \%$ (1). In Thailand, despite the reducing incidence of TB, the reported number of MDR TB cases nearly doubled during 2014-2018 (1); some are likely to be XDR TB. Treatment for patients with DR TB is prolonged and expensive, and outcomes are poor.

Whole-genome sequencing (WGS) of M. tuberculosis provides insights into drug resistance, in which mechanisms almost exclusively involve mutations (mostly single-nucleotide polymorphisms [SNPs], but also insertion/deletions) in genes coding for drug targets or drug-converting enzymes. WGS data can also provide insights into transmission and the dating of clusters (2), in which strains with near-identical genetic variants are likely to be part of a transmission chain (3). Analysis of M. tuberculosis WGS data from isolates across Thailand could provide much-needed insights into MDR/XDR TB transmission. Previous studies of DR TB have used genotyping techniques (e.g., spoligotyping, mycobacterial interspersed repetitive unit-variable-number tandem-repeat, and restriction fragment length polymorphism) $(4,5)$, but these methods have limited resolution for inferring transmission because they investigate $<1 \%$ of the $M$. tuberculosis 
genome. A recent WGS analysis revealed possible clonal transmission of 4 MDR TB isolates in Kanchanaburi Province (6). However, the extent of MDR TB and XDR TB clusters across Thailand is unknown. Our aim was to investigate the clustering patterns and risk factors of possible MDR TB, pre-XDR TB, and XDR TB transmission clusters across Thailand using WGS data.

\section{Methods}

\section{Study Population and Setting}

During 2014-2017, a total of 2,071 M. tuberculosis culture-confirmed MDR TB, pre-XDR TB, and XDR TB cases were listed in the laboratory records of the National Tuberculosis Reference Laboratory (NTRL; Ministry of Public Health) and Siriraj Hospital, Mahidol University, Thailand. These 2 laboratories cover 230 hospitals handling most DR TB cases in Thailand (Appendix 1 Tables 1, 2, https://wwwnc.cdc. gov/EID/article/27/3/20-4364-App1.xlsx) (7). We randomly selected $547 \mathrm{M}$. tuberculosis isolates from MDR TB and pre-XDR TB cases across 6 regions and 71 of 77 provinces nationally. We also included all retrievable $(\mathrm{n}=32) \mathrm{XDR}$ TB isolates (Appendix 1 Table 3). For 11 cases, we used pairs of isolates collected at different times as internal controls for SNP distances. In each control pair, we included the isolate with the most mutations associated with drug resistance or the chronologically earlier isolate in the studied population $(\mathrm{n}=579)$. We retrieved demographic data from laboratory records. The study protocol was approved by the Center for Ethics in Human Research, Khon Kaen University (approval no. HE601249).

\section{Phenotypic Drug-Susceptibility Testing}

We performed phenotypic drug-susceptibility testing (DST) using the standard agar proportional method in Lowenstein-Jensen medium (8). Drug concentrations used were $0.2 \mu \mathrm{g} / \mathrm{mL}$ for isoniazid; $40.0 \mu \mathrm{g} / \mathrm{mL}$ for rifampin, ethionamide, capreomycin, and cycloserine; $2.0 \mu \mathrm{g} / \mathrm{mL}$ for ethambutol, ofloxacin, and levofloxacin; $4.0 \mu \mathrm{g} / \mathrm{mL}$ for streptomycin; $30.0 \mu \mathrm{g} / \mathrm{mL}$ for kanamycin; and $0.5 \mu \mathrm{g} / \mathrm{mL}$ for para-aminosalicylic acid. We used M. tuberculosis H37Rv as the susceptible reference strain.

\section{Whole-Genome Sequence Analysis}

We used multiple loops of $M$. tuberculosis colonies for genomic DNA extraction (with the cetyl-trimethylammonium bromide-sodium chloride method) (9). WGS data for $590 \mathrm{M}$. tuberculosis isolates were produced by NovogeneAIT (https://en.novogene.com) using the HiSeq (Illumina, https://www.illumina. com) platform generating 150-bp paired-end reads. We checked the quality of sequence reads using FastQC version 0.11.7 (10). We mapped high-quality reads from each isolate onto the $\mathrm{H} 37 \mathrm{Rv}$ reference genome (GenBank accession no. NC_000962.3) using BWA-MEM version 0.7.12 (Li H, unpub. data, https:/ / arxiv.org/abs/1303.3997). The average depth of sequencing coverage was high (341.01 \pm 61.98$)$. We used SAMtools version 0.1.19 (11) and GATK version 3.4.0 (12) to call SNPs and insertion/deletions. We filtered variants on the basis of a minimum coverage depth of 10-fold and Q20 minimum base-call quality score, and the intersection set of GATK and SAMtools variants was retained. We used the online tool TB-Profiler version 2.8.6 $(13,14)$ to infer drug resistance and $M$. tuberculosis lineage membership on the basis of SNPs from the WGS data. The WGS data are available in the ENA Sequence Read Archive (https://www. ebi.ac.uk/ena/browser/home) (accession nos. PRJNA598981 and PRJNA613706).

\section{Phylogenetic Analysis}

We constructed a phylogenetic tree based on 26,541 high-confidence SNPs among 590 isolates using the maximum-likelihood method with the selected general time-reversible with gamma-distribution model, implemented within MEGA version 10.1 (15). We excluded the 130 SNPs known to be associated with DR TB found in this study to ensure that they would not affect the phylogenetic analysis. We inferred a bootstrap consensus tree from 1,000 replicates. We produced the phylogenetic tree image using iTOL (16).

\section{Data Analysis}

Isolates forming monophyletic groups in which many or all pairs differed by $\leq 25$ SNPs were placed in the same clade. Clusters included isolates differing by 0-11 SNPs. We regarded members of a single cluster as possibly descended from a single clone through recent transmission. Less-recently transmitted isolates within a clade differed by 12-25 SNPs. We calculated the clustering percentage as (no. clustering isolates/ total no. isolates) $\times 100$. We differentiated isolates with acquired DR TB from possible primary DR TB (MDR TB, pre-XDR TB, and XDR TB) isolates on the basis of acquisition of additional resistance-associated mutations, especially those associated with resistance to fluoroquinolones, kanamycin, or capreomycin, drugs that are used for DR TB classification. For clusters containing isolates with different types of DR TB (such as MDR TB and XDR TB), we used the acquisition of additional drug-resistance SNPs and 
co-ancestral relationships to differentiate between 2 patterns of acquired resistance: chronological (ancestral strain had fewer mutations, lesser type of DR, or both) or nonchronological (ancestral strain had more mutations, stronger type of DR, or both). Although XDR TB and pre-XDR TB could be considered as subsets of MDR TB, we have treated all 3 as separate categories in our analyses.

We analyzed all data using $\mathrm{R}$ statistical software version 3.6.1 (https://www.r-project.org) and considered $\mathrm{p}$ values $<0.05$ to be statistically significant. We analyzed associations between clades/clusters and geography using $\chi^{2}$ tests and visualized them with the R package vcd version 1.4-8. We calculated odds ratios (ORs) with $95 \%$ CIs using the $\mathrm{R}$ package epiR version 1.0-4. We tested factors associated with clustering isolates using the Student $t$-test (numerical data), $\chi^{2}$ test, or Fisher exact test (categorical data), when applicable. We constructed graphs using the $\mathrm{R}$ package ggplot2 version 3.2.1 and built phylo-maps using the package phytools version 0.7-20.

\section{Results}

\section{Study Population and Characteristics}

Most $(466 ; 80.5 \%)$ of the 579 culture-confirmed DR TB cases in the studied population were MDR TB, followed by 81 pre-XDR TB (14.0\%) (Appendix 1 Table 2). We included all available XDR TB isolates $(\mathrm{n}=32)$, constituting $5.5 \%$ of our samples but only $1.5 \%$ of the culture-confirmed 2,071 DR TB isolates collected nationally during 2014-2017. Central and

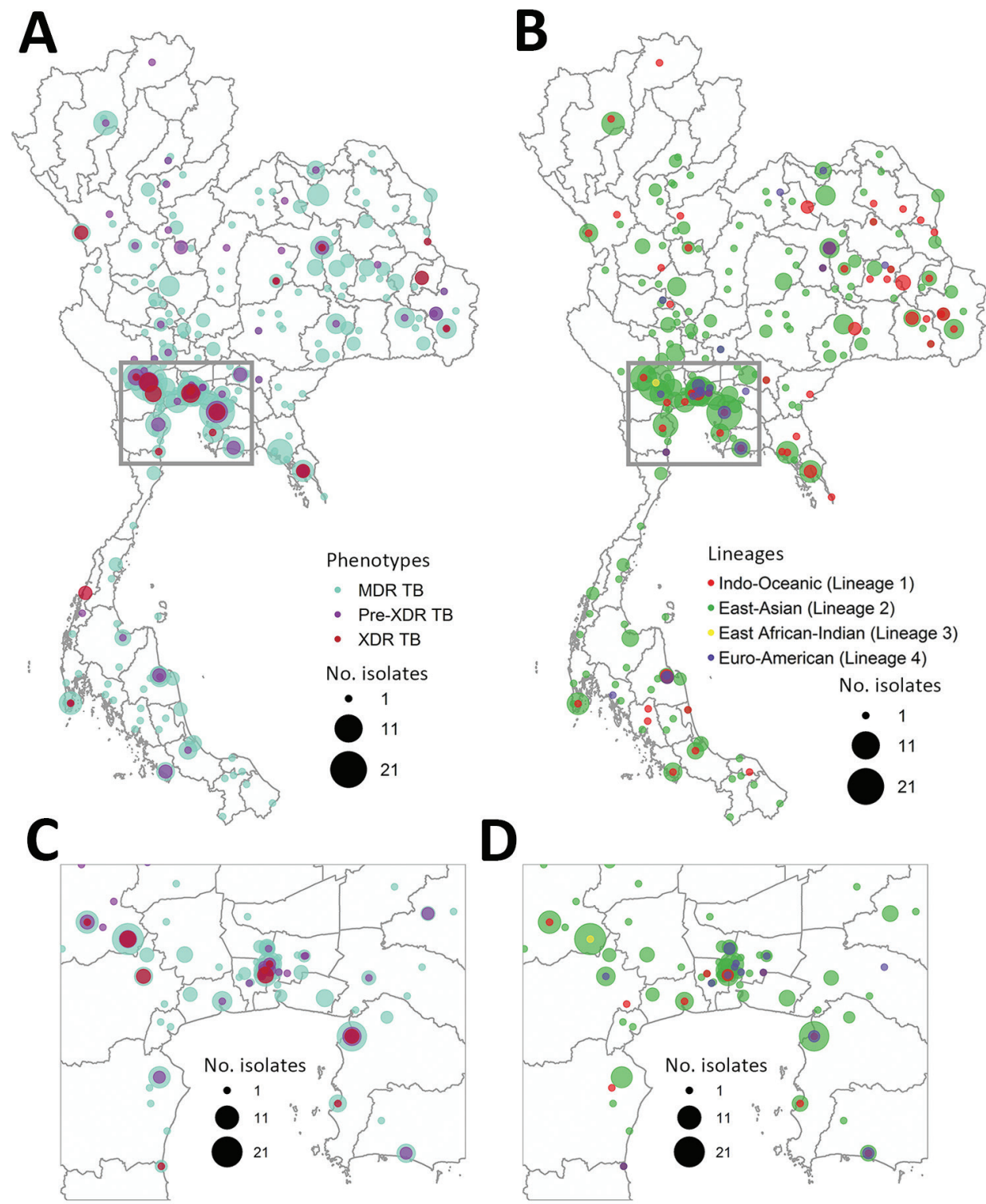

Figure 1. Geographic and lineage distribution of 579 drug-resistant Mycobacterium tuberculosis isolates in Thailand, 2014-2017.

A) Geographic distribution of MDR TB, pre-XDR TB, and XDR TB. B) Lineage distribution of drug-resistant $M$. tuberculosis. C) Drug-resistant types, enlarged from panel A. D) Lineage distribution, enlarged from panel B. The size of each circle is proportional to the number of isolates. MDR, multidrug resistant; TB, tuberculosis; XDR, extensively drug-resistant. 
northeast regions of Thailand had the highest DR TB proportions (Figure 1). The 3 provinces with the highest number of DR TB cases were Bangkok ( $\mathrm{n}=$ $85 ; 14.7 \%)$, Kanchanaburi ( $\mathrm{n}=51 ; 8.8 \%)$, and Chonburi $(\mathrm{n}=37 ; 6.4 \%)$ (Figure 1; Appendix 1 Table 3). Most patients were male $(\mathrm{n}=419 ; 73.1 \%)$ and mean age was $43.5( \pm 14.7)$ years (Appendix 1 Table 4$)$.

\section{Phylogenetic Analysis}

Most of the $M$. tuberculosis isolates belonged to the East-Asian lineage (lineage 2$)(n=482 ; 83.2 \%)$, followed by the Indo-Oceanic lineage (lineage 1$)(n=67$; $11.6 \%)$, the Euro-American lineage (lineage 4$)(n=29$; $5.0 \%)$, and the East African-Indian lineage (lineage 3)
( $\mathrm{n}=1 ; 0.2 \%)$ (Figure 2; Appendix 1 Table 5). Lineage 2.2.1 $(\mathrm{n}=413 ; 71.3 \%)$ was the main sublineage among MDR, pre-XDR, and XDR TB.

\section{Clustering and Possible Transmission Clusters}

The phylogenetic tree (Figure 2) showed enormous diversity among the DR TB isolates from Thailand. Many isolates were distinct, differing from all others at a mean \pm SD of $657 \pm 626$ SNPs. Most isolates ( $\mathrm{n}=319 ; 55.1 \%$ ) grouped into 13 clades, each consisting of 5-86 isolates (Figure 3; Appendix 2 Figure 1, https://wwwnc.cdc. gov/EID/article/27/3/20-4364-App2.pdf). Clades 1, 6,11 , and 13 each consisted of a single small cluster of closely related isolates; the remaining clades included

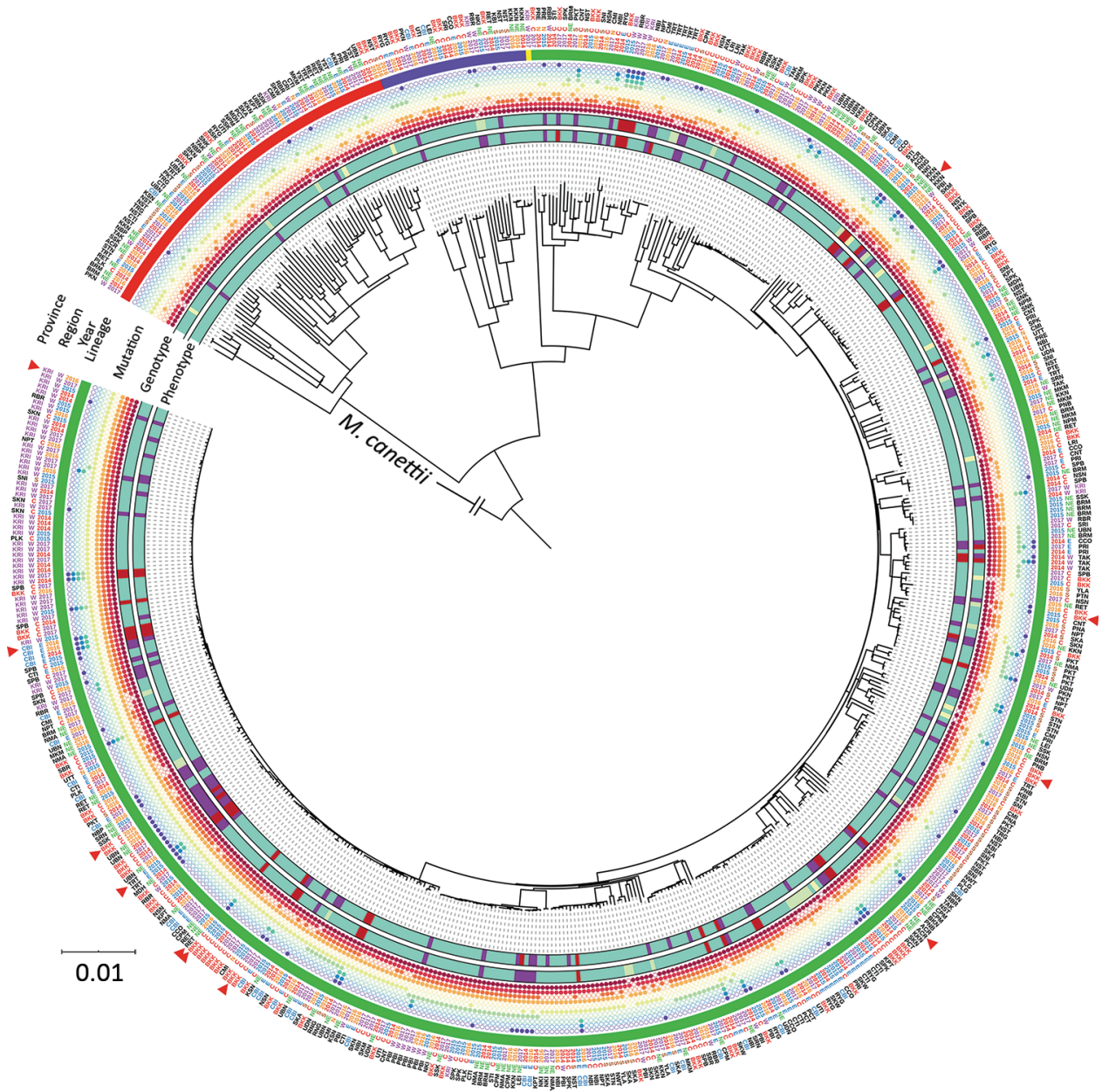

Drug resistance types

\begin{tabular}{l|l} 
Mono-DR TB & Isoniazid \\
Poly-DR TB & $\bigcirc$ Rifampicin \\
MDR TB & $\bigcirc$ Ethambutol \\
Pre-XDR TB & $\bigcirc$ Streptomycin \\
XDR TB & $\bigcirc$ Cycloserine
\end{tabular}

Figure 2. Phylogenetic tree for the 590 drug-resistant Mycobaterium tuberculosis isolates from Thailand, 20142017. From inner to the outer circles: culture-based phenotypic drug-susceptibility test, wholegenome sequencing-based drug-resistance profile (DR TB, MDR TB, pre-XDR TB, and XDR $T B)$, drug-resistance mutations, lineage, year of collection, regions, and provinces. Red triangles indicate the paired isolates from the same patients $(n=11)$. Scale bar indicates the genetic distance proportional to the total number of single nucleotide polymorphisms. $M$. canettii was used as an outgroup. DR TB, drug-resistant tuberculosis; MDR, multidrug resistant; TB, tuberculosis; XDR, extensively drug-resistant. 

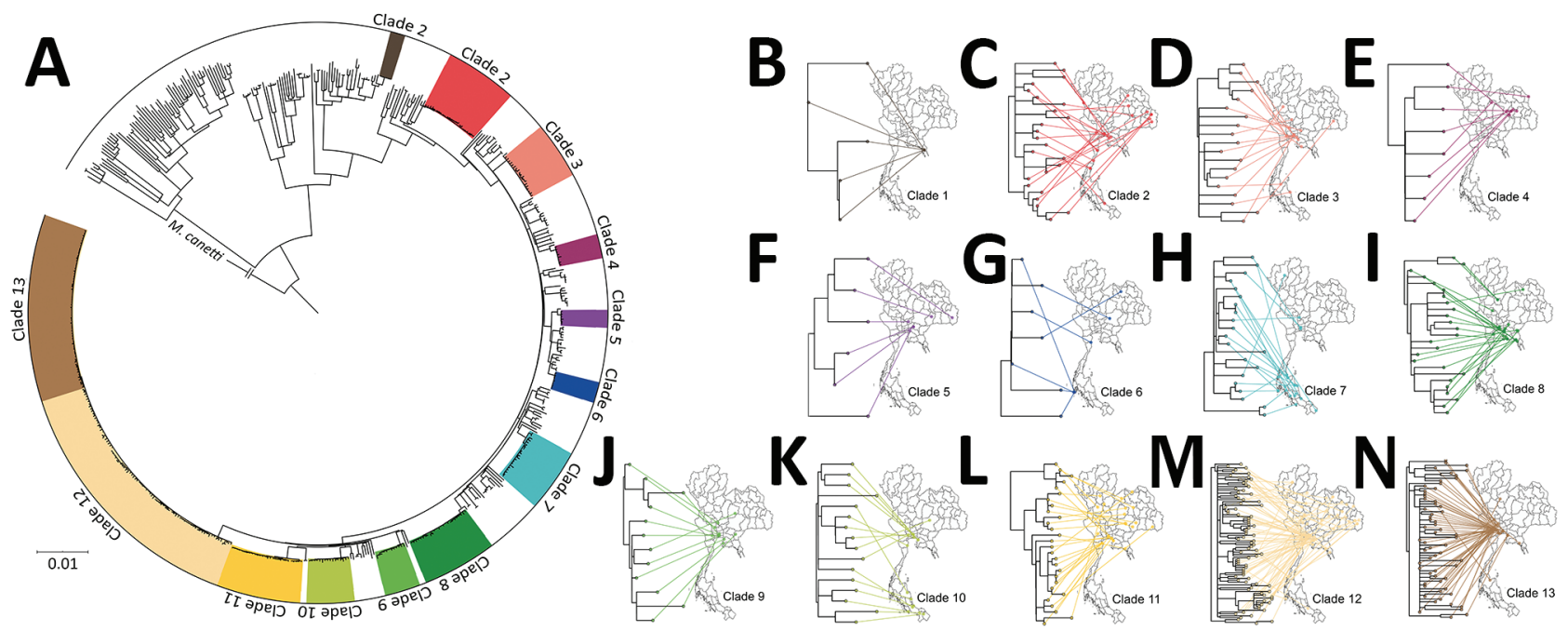

Figure 3. Geographic distribution of 13 major clades of drug-resistant tuberculosis in Thailand. A) The 13 clades are highlighted in the outer circle. Scale bar indicates the genetic distance proportional to the total number of single nucleotide polymorphisms. B-N) Each of the 13 major clades is associated with particular geographic regions, as shown. Mycobacterium canettii was used as an outgroup.

$\geq 1$ possible clusters (Appendix 2 Figure 2). The isolates grouped in each clade were significantly associated with a particular geographic region $(\mathrm{p}<0.001$; Appendix 2 Figure 3, panel A). Clade 1 (Figure 3, panel B) was found only in Trat Province and clade 13 predominated in Kanchanaburi (Figure 3, panel N).

A total of 89 clusters contained 281 isolates (48.5\%) (Appendix 1 Table 6). Sixty clusters (isolates differing by $\leq 11$ SNPs), containing 2-34 isolates, fell within the major clades. A further 29 smaller clusters occurred elsewhere in the tree. Most isolates within a cluster shared geographic links (Figure 4, panels A-F; Appendix 1 Table 6). The percentages of MDR TB, preXDR TB, and XDR TB isolates (based on phenotypic DST) that fell into clusters were $46.1 \%(215 / 466)$ for MDR TB, $49.4 \%$ (40/81) for pre-XDR TB, and $81.3 \%$ (26/32) for XDR TB (Appendix 1 Table 6). Pairwise SNP distances within and between each of the 89 clusters are given summarized (Appendix 1 Table 7).

Some clusters included isolates with different types of DR TB. Nineteen of the 89 clusters (C2, C7, C10, C16, C22, C36, C37, C40, C43, C49, C59, C60, C63, $\mathrm{C} 70, \mathrm{C} 72, \mathrm{C} 76, \mathrm{C} 80, \mathrm{C} 83$, and C89) had a chronological pattern based on the progressive increase in numbers of DR mutations from base to tips in the phylogeny (Appendix 1 Table 8). The pattern of DR mutation changes was nonchronological in clusters C21, C23, C32, C35, C41, C55, C71, and C75. Among the 281 clustering isolates, $81.9 \%$ were classified as possible primary DR TB $(\mathrm{n}=230)$, including MDR TB $(\mathrm{n}=$ $176 / 205 ; 85.9 \%)$, pre-XDR TB $(n=29 / 46 ; 63.0 \%)$, and XDR TB $(\mathrm{n}=14 / 19 ; 73.7 \%)$. In addition, we identified
10 phenotypically MDR isolates and 1 phenotypically pre-XDR TB isolate as possible examples of primary isoniazid resistance $(n=11)$ based on genotypic DR. Other clustering isolates $(\mathrm{n}=51 / 281,18.1 \%)$ exhibited acquired DR TB (MDR TB [n $=29 / 205 ; 14.1 \%]$, preXDR TB $[n=17 / 46 ; 37.0 \%]$, and XDR TB $[n=5 / 19$; 26.3\%]) (Table 1).

Among clustered isolates, there was some discordance between phenotypic DST findings (MDR TB [ $\mathrm{n}=215]$, pre-XDR TB [ $=40]$, and XDR TB [ $\mathrm{n}=$ 26]) and genotypic DST results (poly-DR TB [ $n=11]$, MDR TB [ $\mathrm{n}=205]$, pre-XDR TB $[\mathrm{n}=46]$, and XDR TB $[n=19]$ ) (Appendix 1 Table 8). We identified 11 isolates of phenotypically MDR TB genotypically as poly-DR TB (resistant to $>1$ drug but not to both isoniazid and rifampicin). We identified 66 MDR TB, 9 pre-XDR TB, and 10 XDR TB clusters on the basis of phenotypic DST (Appendix 1 Table 9; Appendix 2 Figure 4, panels A-F). Most pre-XDR TB and XDR TB clusters had hospital-based links (Appendix 1 Table 9). All phenotypic DR TB clusters and resistance types, stratified by province, are shown (Appendix 1 Table 10).

\section{Factors Associated with Possible DR TB Transmission Clusters}

TB patients from whom clustering isolates were obtained had an average age of $\approx 42$ years. Isolates falling within clusters were significantly associated with geographic regions $(p=0.001$; Appendix 2 Figure 3, panel B). Patients with TB who lived in western provinces had a higher risk of being within possible DR 

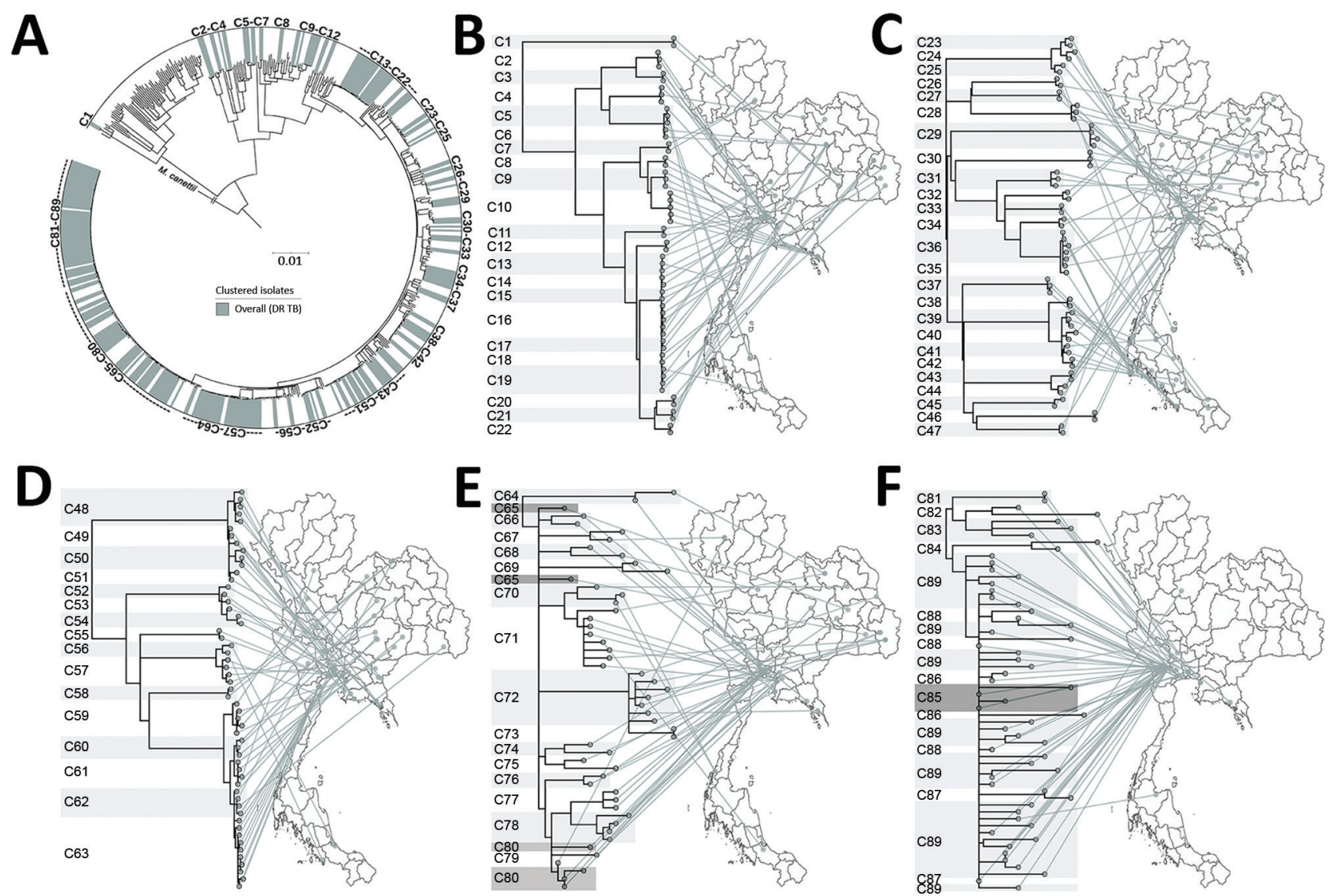

Figure 4. All clusters of DR TB isolates from Thailand. A) A total of 89 clusters are highlighted in the outer circle. Scale bar indicates the genetic distance proportional to the total number of single nucleotide polymorphisms. B-F) Phylogeographical links of each cluster are shown. For clarity, clusters are divided among 5 phylomaps. Some isolates in closely related clusters (C64-C65, C79-C80, and C85-C89) crossed localities. Mycobacterium canettii was used as an outgroup. DR TB, drug-resistant tuberculosis.

TB transmission clusters than those elsewhere (OR 2.44, 95\% CI 1.53-3.89; Table 2). Lineage 2.2.1 (versus other lineages) was associated with a higher risk of possible DR TB transmission clusters (OR 3.59, 95\% CI 2.42-5.32). Lineage 1 had the lowest risk of being represented in DR TB transmission clusters (OR 0.03, 95\% CI 0.01-0.11). Clustering isolates had drug-resistance mutations such as katG S315T, rpoB S450L, and embB G406D (Table 2).

\section{Discussion}

MDR TB and XDR TB are serious global problems, but few studies have focused on their transmission at a nationwide resolution. Thailand has a high burden of MDR TB and increasing numbers of MDR TB cases (1). We sourced 579 DR TB isolates across 71 provinces during 2014-2017. Nearly half of these were in possible transmission clusters, mostly involving $M$. tuberculosis lineage 2.2.1. A total of 89 clusters, most distributed among 13 major clades, contributed to multiclonal MDR TB outbreaks associated with specific regions in Thailand. Bangkok, Kanchanaburi, and Chonburi were the provinces with the highest proportions of MDR TB, pre-XDR TB, and XDR TB clusters (i.e., groups of isolates differing by $\leq 11$ SNPs). We used 2 criteria to select SNP cutoff values. First, the $\leq 11$ SNP difference cutoff for a cluster was derived directly from the maximum number of differences between the 11 paired isolates used as an internal control. Second, we used an SNP cutoff concordant with, or more stringent than, those in previous studies (17-20). Our 11-SNP cutoff was proportionally 0.0004 of the 26,541 SNPs in our total set. This proportion was concordant with that in a previous study (21), and more stringent than those in other studies $(18,20)$. A $<12-S N P$ cutoff has been previously proposed as the upper boundary for possible cluster transmission events (2).

Phylogenetic analysis identified 13 major clades, each associated with a particular region(s). Pairwise 
Table 1. Characteristics of isolates within 89 DR TB clusters, Thailand, 2014-2017*

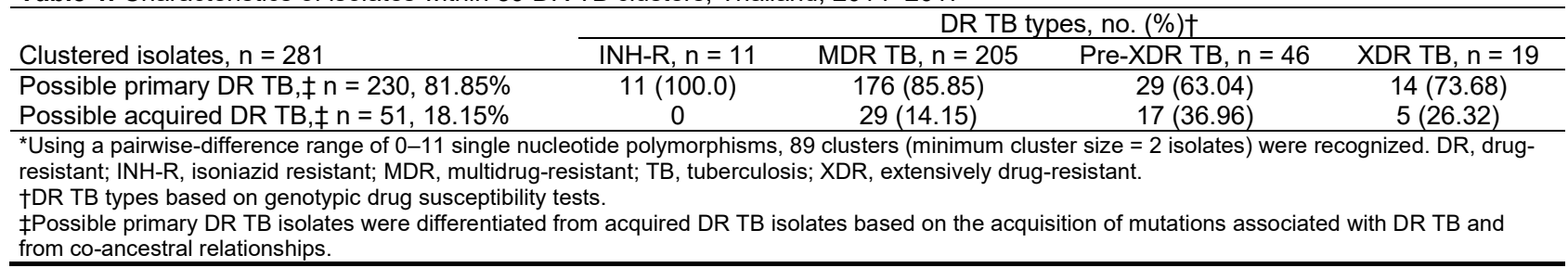

SNP differences between isolates within clades ranged from $>11$ to $\approx 25$, suggesting a range of divergence times from a common ancestor (Appendix 2 Figure 2). On the basis of the transmission time estimates $(0.5$ $\mathrm{SNP} /$ ge-nome/year) for M. tuberculosis (2), some of these major clades might have begun to circulate in Thailand $\approx 20-40$ years ago, others more recently. Isolates differing by 12-25 SNPs nevertheless often shared geographic links. For example, 17 of 21 (81\%) isolates in clade 7 (Figure 3, pan-el H), which had pairwise differences indicating a rela-tively nonrecent common ancestor, were located within

Table 2. Demographic and other factors associated with clustering ( $\leq 11$ SNP difference) of TB isolates, Thailand, 2014-2017* Clustering isolates

\begin{tabular}{|c|c|c|c|c|}
\hline \multirow[b]{2}{*}{ Characteristic } & \multirow[b]{2}{*}{ All isolates, $n=579$} & \multicolumn{2}{|c|}{ Clustering isolates } & \multirow[b]{2}{*}{ Odds ratio $(95 \% \mathrm{Cl})$} \\
\hline & & $\begin{array}{l}\text { Isolates falling within } \\
\text { clusters, } n=281\end{array}$ & $\begin{array}{c}\text { Nonclustering isolates, } \\
n=298\end{array}$ & \\
\hline \multicolumn{5}{|l|}{ Sex, $n=573$} \\
\hline M & $419(73.12)$ & $198(70.71)$ & $221(75.43)$ & $0.79(0.54-1.14)$ \\
\hline $\mathrm{F}$ & $154(26.88)$ & $82(29.29)$ & $72(24.57)$ & $1.27(0.88-1.84)$ \\
\hline \multicolumn{5}{|l|}{ Age, $y, n=508$} \\
\hline Mean \pm SD & $43.51 \pm 14.68$ & $42.02 \pm 15.23$ & $44.94 \pm 14.03$ & NA \\
\hline \multicolumn{5}{|l|}{ Region } \\
\hline Central & $183(31.61)$ & $79(28.11)$ & $104(34.90)$ & $0.73(0.51-1.04)$ \\
\hline Eastern & $88(15.20)$ & $47(16.73)$ & $41(13.76)$ & $1.26(0.80-1.98)$ \\
\hline Northeastern & $125(21.59)$ & $56(19.93)$ & $69(23.15)$ & $0.83(0.56-1.23)$ \\
\hline Northern & $17(2.94)$ & $4(1.42)$ & $13(4.36)$ & $0.32(0.10-0.98)$ \\
\hline Southern & $73(12.61)$ & $33(11.74)$ & $40(13.42)$ & $0.86(0.52-1.40)$ \\
\hline Western & $93(16.06)$ & $62(22.06)$ & $31(10.40)$ & 2.44 (1.53-3.89) \\
\hline \multicolumn{5}{|l|}{ Lineage } \\
\hline 2.1 & $31(5.35)$ & $12(4.27)$ & $19(6.38)$ & $0.66(0.31-1.38)$ \\
\hline 2.2 .1 & $413(71.33)$ & 236 (83.99) & $177(59.40)$ & $3.59(2.42-5.32)$ \\
\hline 2.2.1.1 & $32(5.53)$ & $16(5.69)$ & $16(5.37)$ & $1.06(0.52-2.17)$ \\
\hline 2.2.1.2 and 2.2.2 & $6(1.04)$ & $2(0.71)$ & $4(1.34)$ & $0.53(0.05-3.71)$ \\
\hline 4 & $29(5.01)$ & $13(4.64)$ & $16(5.35)$ & $0.86(0.41-1.82)$ \\
\hline 1 & $67(11.57)$ & $2(0.71)$ & $65(21.81)$ & $0.03(0.01-0.11)$ \\
\hline 3 & $1(0.17)$ & $0(0.00)$ & $1(0.34)$ & NA \\
\hline \multicolumn{5}{|l|}{ Drug-resistance mutations } \\
\hline \multicolumn{5}{|l|}{ Isoniazid, $\mathrm{n}=565$} \\
\hline katG S315T & $448(79.29)$ & $252(89.68)$ & $196(69.01)$ & $3.90(2.46-6.18)$ \\
\hline $\operatorname{inh} A-15 \mathrm{c} / \mathrm{t}$ & $52(9.20)$ & $7(2.49)$ & $45(15.85)$ & $0.14(0.06-0.31)$ \\
\hline \multicolumn{5}{|l|}{ Rifampin, $n=554$} \\
\hline rpoB S450L & $279(50.36)$ & $176(65.19)$ & $103(36.27)$ & $3.29(2.32-4.66)$ \\
\hline \multicolumn{5}{|l|}{ Ethambutol, $n=335$} \\
\hline embB M306V & $85(25.37)$ & $44(20.75)$ & $41(33.33)$ & $0.52(0.32-0.86)$ \\
\hline embB G406D & $66(19.70)$ & $59(27.83)$ & $7(5.69)$ & $6.39(2.81-14.51)$ \\
\hline embB M306I & $56(16.72)$ & $27(12.74)$ & $29(23.58)$ & $0.47(0.26-0.84)$ \\
\hline \multicolumn{5}{|l|}{ Streptomycin, $n=349$} \\
\hline rpsL K43R & $295(84.53)$ & $188(89.95)$ & $107(76.43)$ & $2.76(1.52-5.01)$ \\
\hline \multicolumn{5}{|l|}{ Ethionamide, $\mathrm{n}=268$} \\
\hline ethA 639-640del & $143(53.36)$ & $105(73.43)$ & $38(30.40)$ & $6.33(3.72-10.77)$ \\
\hline $\operatorname{inh} A-15 c / t$ & $65(24.25)$ & $9(6.29)$ & $56(44.80)$ & $0.08(0.04-0.18)$ \\
\hline \multicolumn{5}{|l|}{ Para-aminosalicylic acid, $\mathrm{n}=99$} \\
\hline folC S150G & 39 (39.39) & $32(50.79)$ & $7(19.44)$ & $4.28(1.63-11.19)$ \\
\hline
\end{tabular}

*Values are no. (\%) except as indicated. Bold type indicates statistical significance. NA, not applicable. neighboring provinces of southern Thailand. Clades 1 , 6,11 , and 13 each consisted of isolates differing at very few SNPs, giving us confidence that these were likely examples of recent transmission. Nonetheless, isolates in clade 6 often occurred in different provinces.

The largest and most recent clade was clade 13 (Figure 3, panel N), comprising 62 cases (46 MDR TB, 11 pre-XDR TB, and 5 XDR TB based on phenotypic DST) found in the western region, especially in Kanchanaburi. This finding suggests that clones of pre-XDR TB and XDR TB may emerge from recent 
MDR TB ancestors. We confirmed a previous report (22) that there was a large MDR TB outbreak in Kanchanaburi. In addition, clade 13 is sister to clade 12, which consists of strains that spread in both central (especially Bangkok) and northeast Thailand and contain less recently transmitted strains. Therefore, the MDR TB outbreak clade in Kanchanaburi was derived from a less recently transmitted clade elsewhere in Thailand.

We identified 89 clusters (isolates in each differing by $\leq 11$ SNPs) of DR TB in Thailand. The clustered isolates showed a strong association with geographic region. The largest cluster (C89), within clade 13 in Kanchanaburi, comprised 34 isolates (27 MDR TB and 7 pre-XDR TB based on phenotypic DST). In South Africa, WGS analysis of a large XDR TB cohort (>400 cases) from a single province showed that only $30 \%$ of participants had clear epidemiologic links (person-person or hospital link): $70 \%$ of transmission events may have resulted from casual contact between persons not known to one another (23). Another study in South Africa showed that $19 \%$ of XDR TB patients discharged from the hospital caused secondary XDR TB cases in the community (24). Here, we found 9 clusters of pre-XDR TB (the largest with 7 isolates) and 10 clusters (the largest with 4 isolates) of XDR TB in Thailand (Appendix 1 Table 9; Appendix 2 Figure 4).

To reflect the extent of the DR TB outbreak in Thailand, we calculated the proportion of isolates falling into the 89 DR TB clusters (Table 1). In some clusters, isolates exhibited different types of DR TB associated with chronology, revealing the progression of DR mutations in the phylogeny, moving from the ancestor toward the tips of the tree (Appendix 1 Table 8). Based on mutation-acquisition analysis within this phylogeny, we saw examples of possible primary resistance in $85.9 \%$ of MDR TB, $63.0 \%$ of preXDR TB, and $73.7 \%$ of XDR TB cases. Eight clusters included isolates with different types of DR and more resistance-associated mutations in the ancestral strain than in its descendants. This situation might be explained by different durations of the latency stage occurring after transmission events leading to the emergence of less troublesome DR TB cases (such as MDR TB) later than the more troublesome cases (such as XDR TB) (25). Because not all cases from the possible transmission chain could be included, undetected primary resistance might exist. Data from all DR TB cases in the community and information on treatment history and known exposure are needed to accurately and completely estimate the extent of primary DR TB. The proportion of primary DR TB cases could be higher because we reported numbers of MDR TB cases excluding pre-XDR TB and XDR TB (each of which was reported as a separate subset). In addition, some index cases might not have been included in the selected population.

Previously reported factors contributing to MDR TB transmission include illicit drug usage (26); delayed TB diagnosis and being $>45$ years of age (18); and being single, having low income, suffering frequent stress and other diseases, and lacking medical insurance (27). Lineage 2 predominated in previous studies of transmission of MDR TB $(18,26,28)$. We found that infection with lineage 2.2.1 is the strongest predictor (3.6-fold) of DR TB clusters, whereas infection with lineage 1 had the lowest risk. Living in the western region of Thailand increased the risk of being in DR TB clusters by 2.4-fold. The western region, being close to the border with Myanmar, differs from other regions of the country in terms of both ethnicity and economic development. These differences might explain the increased risk there (29). Previously, clustering isolates were more likely to have mutations of rpoB S450L $(18,30)$, katG S315T, or the inhA promoter (31). We also found a pattern of drug resistance-associated mutations (katG S315T, rpoB S450L, embB G406D, rpsL K43R, ethA 639-640del, and folC S150G) in clusters.

The DR TB situation in Thailand is a major concern and requires urgent implementation of control measures such as active case finding to disrupt the transmission chain and targeted intervention and contact tracing in hotspot regions. The mortality rate and cost of treatment of XDR TB is very high (32); therefore, these DR types should be the priority for intervention. The large size of some clusters might reflect their high transmissibility (33); thus, tracking clade 13 at Kanchanaburi should be a priority. Besides the 13 major clades, several small clusters of DR TB were found in many provinces. The potential for expansion of these small clusters is unknown. Here, we also identified the hotspot provinces to help prioritize locations for intervention.

Globally, few studies at the nationwide scale have used WGS analysis of MDR TB, pre-XDR TB, and XDR TB $(26,30,34-36)$. Older studies have used blunt genotyping tools (e.g., IS6110 restriction fragment length polymorphism, spoligotyping, and mycobacterial interspersed repetitive unit-variable-number tandem-repeat) with limited or convenient sample sizes. DR TB studies using WGS in Saudi Arabia and Portugal have revealed transmission clusters of MDR TB; however, they had small samples and provided limited data on epidemiologic links $(36,37)$. Extrapolating from our 
findings, primary-resistant TB strains may be the main contributors to the current global problem of high MDR TB and XDR TB prevalence.

The primary limitations of our study were that it was retrospective rather than prospective, lacked socioeconomic data for analysis and lacked fine-scale data of epidemiologic links: possible transmission clusters were presumed only from the genetic distances among isolates and each patient's hospital and province of residence. In addition, an accurate estimation of the exact time of the possible transmission cannot be made: clusters originating years ago may be continuing to spread. We also lacked information about treatment and exposure history and of the complete population to identify all index cases to differentiate between primary and acquired DR TB. In addition, the prevalence and clustering of MDR TB, pre-XDR TB, and XDR TB isolates in some provinces might be underestimated because of the low coverage of DST for the first-line drugs among TB cases (1).

In conclusion, we have demonstrated the usefulness of WGS for DR TB epidemiology. We have shown that close to half of MDR TB, pre-XDR TB, and XDR TB cases in Thailand might be caused by transmission clusters. Two thirds of pre-XDR TB and three quarters of MDR TB and XDR TB clustering isolates were possible examples of primary resistance. These results indicate that the emergence of MDR TB, pre-XDR TB, and XDR TB cases in Thailand might be from a narrow base of ancestral strains. The high prevalence of MDR/XDR TB in Thailand might be the result of multiclonal outbreaks. People living in the western region of Thailand had a 2.4-fold increased risk of DR TB clusters, and lineage 2.2.1 conferred a 3.6-fold increased risk of forming DR TB clusters relative to other lineages.

\section{Acknowledgments}

We thank the Research and Diagnostic Center for Emerging and Infectious Diseases (RCEID), Khon Kaen University, for laboratory and research facility support.

This work was supported by the National Research Council of Thailand and Health System Research Institute (HSRI) (grant nos. 60-057 and 62-003 to K.F.). D.N. is funded by Faculty of Medicine, Khon Kaen University. T.G.C. is funded by the Medical Research Council UK (grant nos. MR/M01360X/1, MR/N010469/1, MR/R025576/1, and MR/R020973/1) and Biotechnology and Biological Sciences Research Council UK (BB/R013063/1). A.C. is funded by the Medical Research Council and National Science and Technology Development Agency (MRC/NSTDA) (grant no. P-18-50228).
This work is dedicated to the late HRH Princess Galyanivadhana, the patroness of the Drug-Resistant Tuberculosis Fund.

\section{About the Author}

Mr. Nonghanphithak is a PhD student in the Medical Microbiology Program, Faculty of Medicine, Khon Kaen University, Khon Kaen, Thailand. His research interest is in the molecular epidemiology and prediction of drug resistance in tuberculosis using whole-genome sequencing techniques.

\section{References}

1. World Health Organization. Global tuberculosis report 2019. Geneva: The Organization; 2019.

2. Meehan CJ, Moris P, Kohl TA, Pečerska J, Akter S, Merker M, et al. The relationship between transmission time and clustering methods in Mycobacterium tuberculosis epidemiology. EBioMedicine. 2018;37:410-6. https:/ / doi.org/ 10.1016/j.ebiom.2018.10.013

3. Guerra-Assunção JA, Crampin AC, Houben RM, Mzembe T, Mallard K, Coll F, et al. Large-scale whole genome sequencing of $M$. tuberculosis provides insights into transmission in a high prevalence area. eLife. 2015;4:e05166. https://doi.org/10.7554/eLife.05166

4. Disratthakit A, Meada S, Prammananan T, Thaipisuttikul I, Doi N, Chaiprasert A. Genotypic diversity of multidrug-, quinolone- and extensively drug-resistant Mycobacterium tuberculosis isolates in Thailand. Infect Genet Evol. 2015;32:432-9. https:// doi.org/10.1016/j.meegid.2015.03.038

5. Rienthong D, Ajawatanawong P, Rienthong S, Smithtikarn S, Akarasewi P, Chaiprasert A, et al. Restriction fragment length polymorphism study of nationwide samples of Mycobacterium tuberculosis in Thailand, 1997-1998. Int J Tuberc Lung Dis. 2005;9:576-81.

6. Regmi SM, Chaiprasert A, Kulawonganunchai S, Tongsima S, Coker OO, Prammananan T, et al. Whole genome sequence analysis of multidrug-resistant Mycobacterium tuberculosis Beijing isolates from an outbreak in Thailand. Mol Genet Genomics. 2015;290:1933-41. https://doi.org/10.1007/s00438-015-1048-0

7. World Health Organization. Global tuberculosis report 2018. Geneva: The Organization; 2018.

8. Canetti G, Fox W, Khomenko A, Mahler HT, Menon NK, Mitchison DA, et al. Advances in techniques of testing mycobacterial drug sensitivity, and the use of sensitivity tests in tuberculosis control programmes. Bull World Health Organ. 1969;41:21-43.

9. Larsen MH, Biermann K, Tandberg S, Hsu T, Jacobs WR Jr. Genetic manipulation of Mycobacterium tuberculosis. Curr Protoc Microbiol. 2007;6:10A.2.1-21. https:// doi.org/ 10.1002/9780471729259.mc10a02s6

10. Andrews S. FastQC: A quality control tool for high throughput sequence data. Cambridge (UK): Babraham Bioinformatics; 2010.

11. Li H, Handsaker B, Wysoker A, Fennell T, Ruan J, Homer N, et al.; 1000 Genome Project Data Processing Subgroup. The Sequence Alignment/Map format and SAMtools. Bioinformatics. 2009;25:2078-9. https://doi.org/10.1093/ bioinformatics/btp352

12. McKenna A, Hanna M, Banks E, Sivachenko A, Cibulskis K, Kernytsky A, et al. The Genome Analysis Toolkit: a MapReduce framework for analyzing next-generation DNA 
sequencing data. Genome Res. 2010;20:1297-303. https://doi.org/10.1101/gr.107524.110

13. Coll F, McNerney R, Preston MD, Guerra-Assunção JA, Warry A, Hill-Cawthorne G, et al. Rapid determination of anti-tuberculosis drug resistance from whole-genome sequences. Genome Med. 2015;7:51. https://doi.org/ 10.1186/s13073-015-0164-0

14. Phelan JE, O'Sullivan DM, Machado D, Ramos J, Oppong YEA, Campino S, et al. Integrating informatics tools and portable sequencing technology for rapid detection of resistance to anti-tuberculous drugs. Genome Med. 2019;11:41. https://doi.org/10.1186/s13073-019-0650-x

15. Kumar S, Stecher G, Li M, Knyaz C, Tamura K. MEGA X: Molecular Evolutionary Genetics Analysis across computing platforms. Mol Biol Evol. 2018;35:1547-9. https://doi.org/10.1093/molbev/msy096

16. Letunic I, Bork P. Interactive Tree Of Life (iTOL) v4: recent updates and new developments. Nucleic Acids Res. 2019;47(W1):W256-9. https://doi.org/10.1093/nar/gkz239

17. Walker TM, Ip CL, Harrell RH, Evans JT, Kapatai G, Dedicoat MJ, et al. Whole-genome sequencing to delineate Mycobacterium tuberculosis outbreaks: a retrospective observational study. Lancet Infect Dis. 2013;13:137-46. https://doi.org/10.1016/S1473-3099(12)70277-3

18. Yang C, Luo T, Shen X, Wu J, Gan M, Xu P, et al. Transmission of multidrug-resistant Mycobacterium tuberculosis in Shanghai, China: a retrospective observational study using whole-genome sequencing and epidemiological investigation. Lancet Infect Dis. 2017;17:275-84. https://doi.org/10.1016/S1473-3099(16)30418-2

19. Xu Y, Cancino-Muñoz I, Torres-Puente M, Villamayor LM, Borrás R, Borrás-Máñez M, et al. High-resolution mapping of tuberculosis transmission: whole genome sequencing and phylogenetic modelling of a cohort from Valencia region, Spain. PLoS Med. 2019;16:e1002961. https:/ / doi.org/ 10.1371/journal.pmed.1002961

20. Shuaib YA, Khalil EAG, Wieler LH, Schaible UE, Bakheit MA, Mohamed-Noor SE, et al. Mycobacterium tuberculosis complex lineage 3 as causative agent of pulmonary tuberculosis, eastern Sudan. Emerg Infect Dis. 2020;26:42736. https:// doi.org/10.3201/eid2603.191145

21. Merker M, Nikolaevskaya E, Kohl TA, Molina-Moya B, Pavlovska O, Brännberg P, et al. Multidrug- and extensively drug-resistant Mycobacterium tuberculosis Beijing clades, Ukraine, 2015. Emerg Infect Dis. 2020;26:481-90. https:// doi.org/10.3201/eid2603.190525

22. Boonthanapat N, Soontornmon K, Pungrassami P, Sukhasitwanichkul J, Mahasirimongkol S, Jiraphongsa C, et al. Use of network analysis multidrug-resistant tuberculosis contact investigation in Kanchanaburi, Thailand. Trop Med Int Health. 2019;24:320-7. https:/ / doi.org/10.1111/tmi.13190

23. Auld SC, Shah NS, Mathema B, Brown TS, Ismail N, Omar SV, et al. Extensively drug-resistant tuberculosis in South Africa: genomic evidence supporting transmission in communities. Eur Respir J. 2018;52:1800246. https:/ / doi.org/10.1183/13993003.00246-2018

24. Dheda K, Limberis JD, Pietersen E, Phelan J, Esmail A, Lesosky M, et al. Outcomes, infectiousness, and transmission dynamics of patients with extensively drug-resistant tuberculosis and home-discharged patients with programmatically incurable tuberculosis: a prospective cohort study. Lancet Respir Med. 2017;5:269-81. https:/ / doi.org/10.1016/S2213-2600(16)30433-7

25. Knight GM, McQuaid CF, Dodd PJ, Houben RMGJ. Global burden of latent multidrug-resistant tuberculosis: trends and estimates based on mathematical modelling. Lancet
Infect Dis. 2019;19:903-12. https:/ / doi.org/10.1016/S14733099(19)30307-X

26. Anderson LF, Tamne S, Brown T, Watson JP, Mullarkey C, Zenner D, et al. Transmission of multidrug-resistant tuberculosis in the UK: a cross-sectional molecular and epidemiological study of clustering and contact tracing. Lancet Infect Dis. 2014;14:406-15. https:/ / doi.org/10.1016/ S1473-3099(14)70022-2

27. Li WB, Zhang YQ, Xing J, Ma ZY, Qu YH, Li XX. Factors associated with primary transmission of multidrug-resistant tuberculosis compared with healthy controls in Henan Province, China. Infect Dis Poverty. 2015;4:14. https://doi.org/10.1186/s40249-015-0045-1

28. Marais BJ, Mlambo CK, Rastogi N, Zozio T, Duse AG, Victor TC, et al. Epidemic spread of multidrug-resistant tuberculosis in Johannesburg, South Africa. J Clin Microbiol. 2013;51:1818-25. https:// doi.org/10.1128/JCM.00200-13

29. Palittapongarnpim $P$, Ajawatanawong $P$, Viratyosin $W$, Smittipat N, Disratthakit A, Mahasirimongkol S, et al. Evidence for host-bacterial co-evolution via genome sequence analysis of 480 Thai Mycobacterium tuberculosis lineage 1 isolates. Sci Rep. 2018;8:11597. https://doi.org/10.1038/s41598-018-29986-3

30. Wang SF, Zhou Y, Pang Y, Zheng HW, Zhao YL. Prevalence and risk factors of primary drug-resistant tuberculosis in China. Biomed Environ Sci. 2016;29:91-8. https:/ / doi.org/ 10.3967/bes2016.010

31. Gagneux S, Burgos MV, DeRiemer K, Enciso A, Muñoz S, Hopewell PC, et al. Impact of bacterial genetics on the transmission of isoniazid-resistant Mycobacterium tuberculosis. PLoS Pathog. 2006;2:e61. https://doi.org/10.1371/journal. ppat.0020061

32. Manjelievskaia J, Erck D, Piracha S, Schrager L. Drugresistant TB: deadly, costly and in need of a vaccine. Trans $R$ Soc Trop Med Hyg. 2016;110:186-91. https:/ / doi.org/10.1093/trstmh/trw006

33. Sobkowiak B, Banda L, Mzembe T, Crampin AC, Glynn JR, Clark TG. Bayesian reconstruction of Mycobacterium tuberculosis transmission networks in a high incidence area over two decades in Malawi reveals associated risk factors and genomic variants. Microb Genom. 2020;6:e00361. https://doi.org/10.1099/mgen.0.000361

34. Jagielski T, Brzostek A, van Belkum A, Dziadek J, Augustynowicz-Kopeć E, Zwolska Z. A close-up on the epidemiology and transmission of multidrug-resistant tuberculosis in Poland. Eur J Clin Microbiol Infect Dis. 2015;34:41-53. https:// doi.org/10.1007/s10096-014-2202-z

35. Somoskovi A, Helbling P, Deggim V, Hömke R, Ritter C, Böttger EC. Transmission of multidrug-resistant tuberculosis in a low-incidence setting, Switzerland, 2006 to 2012. Euro Surveill. 2014;19:20736. https:// doi.org/10.2807/1560-7917. ES2014.19.11.20736

36. Oliveira O, Gaio R, Carvalho C, Correia-Neves M, Duarte R, Rito T. A nationwide study of multidrug-resistant tuberculosis in Portugal 2014-2017 using epidemiological and molecular clustering analyses. BMC Infect Dis. 2019;19:567. https:// doi.org/10.1186/s12879-019-4189-7

37. Al-Ghafli H, Kohl TA, Merker M, Varghese B, Halees A, Niemann S, et al. Drug-resistance profiling and transmission dynamics of multidrug-resistant Mycobacterium tuberculosis in Saudi Arabia revealed by whole genome sequencing. Infect Drug Resist. 2018;11:2219-29. https://doi.org/10.2147/IDR. S181124

Address for correspondence: Kiatichai Faksri, Department of Microbiology, Faculty of Medicine, Khon Kaen University, Khon Kaen 40002, Thailand; email: kiatichai@kku.ac.th 